\title{
Total Body PET Imaging From Mice to Humans
}

\author{
Terry Jones* \\ Department of Radiology, Medical Center, University of California, Davis, Sacramento, CA, United States
}

The technology of Total Body PET scanning has been recently established. To advance its application for undertaking across body research in humans, small animal PET scanning is positioned to undertake programs of the pre-clinical development of paradigms and protocols that would be translatable to human total body studies.

Keywords: positron emission tomography, total body PET, small animal PET, systems biology, molecular imaging

\section{INTRODUCTION}

Positron emission tomography (PET) is the most specific and sensitive method for imaging regional tissue molecular interactions and pathways in humans [1]. The specificity comes from the range of imaging biomarkers that can be used labeled with positron emitting radionuclides. These range from the short lived of minutes radioactive half-life, to longer lived of hours to days half-life. The radiochemists have overcome the challenges of radiosyntheses labeling with radionuclides such as carbon-11 and fluorine-18 to chelation radio labeling with longer lived metals such as zirconium-89. The sensitivity for detecting low mass, i.e., truly tracer levels of biomarkers arises through:

OPEN ACCESS

Edited by:

Claudia Kuntner,

Austrian Institute of Technology

(AIT), Austria

Reviewed by:

Daniele De Paula Faria,

University of São Paulo, Brazil

Timothy DeGrado,

Mayo Clinic, United States

*Correspondence:

Terry Jones

terry.jones40@googlemail.com

Specialty section:

This article was submitted to

Medical Physics and Imaging,

a section of the journal

Frontiers in Physics

Received: 16 December 2019

Accepted: 04 March 2020

Published: 03 April 2020

Citation:

Jones T (2020) Total Body PET

Imaging From Mice to Humans.

Front. Phys. 8:77.

doi: 10.3389/fphy.2020.00077 i) radiolabeling procedures that provide high specific activity of the tracer compound,

ii) the use of imaging based upon coincident detection which offers highly efficient, electronic collimation for recording regional tissue concentrations of tracer.

Exploiting the electronic collimation through 3D PET by extending the axial length of PET scanners, evolved cautiously in the early years. This was initially restrained both by cost and ensuring the important need to record quantitative scan data that can be processed to derive meaningful quantitative parameters of regional tissue function. Initial clinical research and healthcare applications of PET focused on individual organs of the body e.g., brain or heart and oncology focused on static $\left[{ }^{18} \mathrm{~F}\right]$ FDG imaging for tumor detection within the torso. Hence, there was little immediate incentive to embrace the cost of extending the axial length of PET scanners beyond a typical $24 \mathrm{~cm}$ axial length. For a review of the development of human PET scanners see Jones and Townsend [2].

\section{SMALL ANIMAL PET SCANNERS}

Preclinical small animal PET scanners arose both from the need to support the preclinical development of imaging biomarkers and the advances initially made to maximize the use of electronic collimation in humans. It was realized that the efficiency offered by wide axial coincidence detection resulted in the sensitivity needed to achieve high spatial resolution when scanning small laboratory animals. Since the early 1990's, small animal PET scanners have undergone a number of technical advances in order to improve the spatial resolution along with the accompanying sensitivity to support the statistical demands of increasing the spatial resolution see Miyaoka and Lehnert [3]. Total body imaging in mice is well-established as most of the preclinical scanners provide axial field 
of views covering the whole body of mice. As such in preclinical research it is common to examine radiotracer distribution at a whole-body level in mice. Moreover, in preclinical imaging, researchers have also profited from dynamic imaging to obtain quantitative results by kinetic modeling $[4,5]$. However, in human imaging the first total body scanner became available in 2018 and total body based kinetic modeling still in its infancies.

\section{HUMAN TOTAL BODY PET SCANNER (TBP)}

A human total body PET scanner (TBP) of some near 2 meters axial length has been developed that is destined to become transformative for molecular imaging in humans [6-8]. The first human results have been obtained demonstrating; images of high statistical quality, total body regional kinetics which, when processed, produce total body quantitative parametric images of tissue function $[8,9]$ as shown in Figures 1, 2. An alternative design of TBP scanner, using non-Anger logic detectors, is under construction with a projected overall length of 1.4 meters $[10,11]$.

The projected applications of TBP are in both clinical healthcare and research with iteration between both areas.

\section{Clinical Healthcare Procedures With TBP}

In standard clinical healthcare, TBP will enable current examinations to be undertaken much better with advantages in quality and practicability:

A. The quality advantages of TBP are:

i) Significantly improved image quality,

ii) Quantitative imaging, based upon kinetic modeling is much more easily, and non-invasively undertaken using high quality image derived arterial input functions,

iii) Widening the applications of PET — translating from the new clinical research areas developed with TBP,

iv) Increased range of imaging bio-markers available in a given imaging center, from wider and further afield commercial distribution centers because of longer effective shelf lives.

B. The practical advantages of TBP in healthcare are:

i) Scan times of minutes: more patient throughput per unit of time,

ii) Scan times of minutes resulting in less movement induced blurring,

iii) Remove the need for arterial blood sampling,

iv) Could do the clinical load of 3-4 conventional scanners: space and staff saving,

v) Prescribe scans with lower radiation dose to patients and staff, and offering opportunities for screening,

\section{(a)}
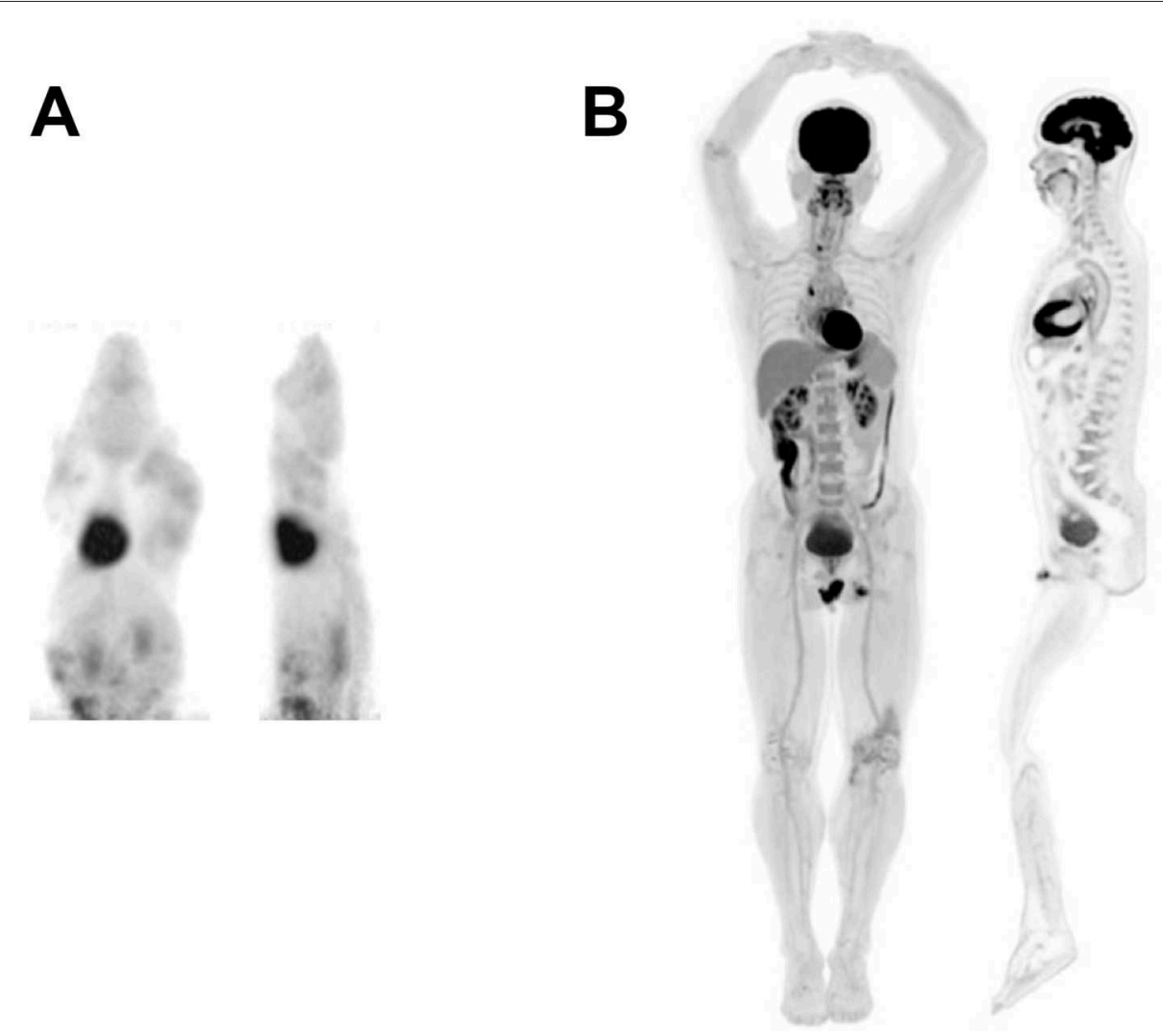

FIGURE 1 | Selected views from a (A) mouse and (B) human total-body scan showing total-body MIP and total-body sagittal view. Mouse image was acquired using the micro PET Focus 220 scanner whereas the human image was acquired using the EXPLORER total body PET scanner. This research was originally published in JNM [8]. () SNMMI. 


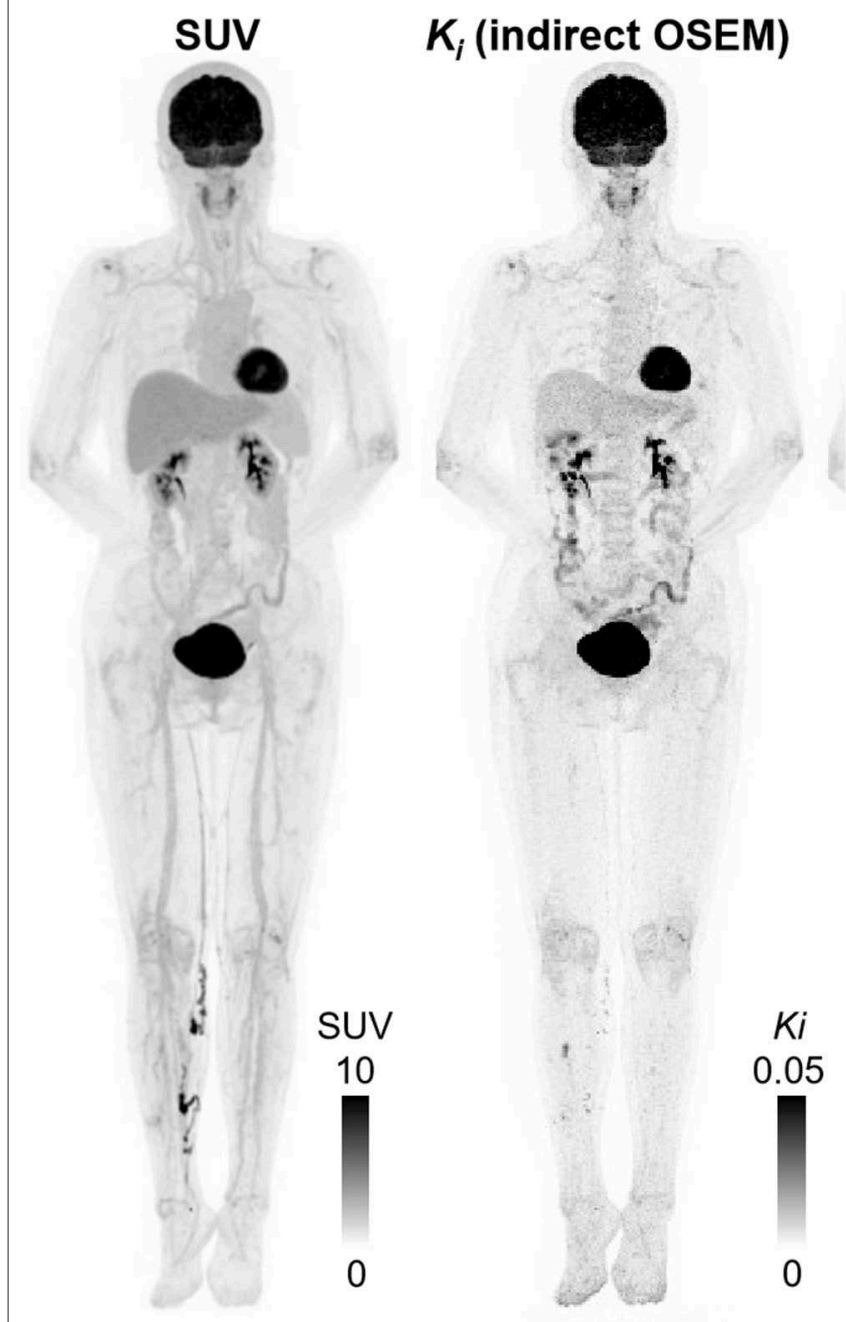

FIGURE 2 | Maximum intensity projection (MIP) using last 30 min of data (left to right): SUV; indirect OSEM Patlak slope $K_{i}$ (3 iterations 10 subsets); This research was originally published in JNM [9]. ๑ SNMMI.

vi) Longer shelf life of radiolabelled tracers from distribution centers-avoiding the cost of in-house GMP production of new tracers labeled with fluorine-18, and, across cities, carbon-11.

\section{Clinical Research Applications With TBP}

The clinical research applications with TBP rests to some extent on the underlying means to study lower levels of occult pathology e.g., in cancer, inflammation and infection but also the "Systems Biology" of human beings. Such studies are destined to cover: pharmacokinetics, pharmacodynamics, oncology, cardiovascular, endocrinology, immunology, maternal-fetal studies, and brain-body interactions e.g., in psychiatry, neurology, and inflammatory diseases.

\section{OUTLOOK ON TBP IMAGING}

From the outset, the take up of TBP is facing numbers of challenges. In the first instance this is the cost of such an instrument. However, "Since the 1970's, the technical developments in PET instrumentation have resulted in major improvements in image quality for patient studies. These advances, although initially seen to be somewhat costly, have, by opening up new clinical applications, proved ultimately to be cost-effective" [2]. Never-the-less, funders at this time are asking for proof-of-concept pilot data that demonstrates that transformative studies will be made possible. Hence the pressure on the few operational TBP scanners to provide for early proofof-concept data. To advance the new investigative field of total body PET, it will be necessary to formulate, validate/characterize, suitable paradigms and corresponding protocols. TBP based "systems biology" research is in its infancy. Such research would cover for example measures of: The whole-body's functioning of the vascular system and regional variations and changes therein. Physiological and endocrinology interactions between different organs of the body. Movement of cells from within and across the body. Pharmacokinetics and pharmacodynamic effects throughout the body. Physiological and metabolic interaction and exchange within and between the mother and fetus.

While some pilot human studies are envisaged, in order, to address the broad scope for such investigations, there is clearly a need to explore and consolidate such pilots at the pre-clinical level. This is where small animal PET scanner technology, which is already in place for total body PET studies of mice and rats, is destined to play a significant role in establishing paradigms and protocols that are translatable to human TBP. It is estimated that there are $\sim 400$ small animal PET scanners worldwide. These already existing extensive preclinical small animal PET programs represent a major resource within the molecular imaging community. They are wellpositioned to develop, characterize and validate the paradigms and protocols for the more advanced total body PET scanning based "system biology" human studies. Inevitably there will be some reverse translation not least asking for more performance of the small animal PET technology and effecting quantitative parametric imaging.

\section{ETHICS STATEMENT}

The study protocol was approved by the Zhongshan Hospital Shanghai Ethics Committee. The patients/participants provided their written informed consent to participate in this study.

\section{AUTHOR CONTRIBUTIONS}

The author confirms being the sole contributor of this work and has approved it for publication.

\section{FUNDING}

The EXPLORER scanner was developed with support from NCI, NIBIB, and the NIH Office of the Director through Transformative R01 CA 206187, with additional support from United Imaging Healthcare. 


\section{ACKNOWLEDGMENTS}

The author acknowledges the generous invitation to join in the development of the EXPLORER Total Body PET scanner project received from Simon Cherry and

\section{REFERENCES}

1. Jones T. The role of positron emission tomography within the spectrum of medical imaging. Eur J Nucl Med. (1996) 23:207-11. doi: 10.1007/BF01731847

2. Jones $\mathrm{T}$, Townsend D. History and future technical innovation in positron emission tomography. J Med Imaging. (2017) 4:011013. doi: 10.1117/1.JMI.4.1.011013

3. Miyaoka RS, Lehnert AL. Small Animal PET: Review of what have we done and where we are going. Phys Med Biol. (in press).

4. Dupont P, Warwick J. Kinetic modelling in small animal imaging with PET. Methods. (2009) 48:98-103. doi: 10.1016/j.ymeth.2009.03.008

5. Zheng X, Wen L, Yu SJ, Huang SC, Feng DD. A study of non-invasive Patlak quantification for whole-body dynamic FDG-PET studies of mice. Biomed Signal Process Control. (2012) 7:438-46. doi: 10.1016/j.bspc.2011.11.005

6. Cherry SR, Badawi RD, Karp JS, Moses WW, Price P, Jones T. Total-body imaging: Transforming the role of positron emission tomography. Sci Transl Med. (2017) 9:eaaf6169. doi: 10.1126/scitranslmed.aaf6169

7. Cherry SR, Jones T, Karp JS, Qi J, Moses WW, Badawi RD. Total-body PET: maximizing sensitivity to create new opportunities for clinical research and patient care. J Nucl Med. (2018) 59:3-12. doi: 10.2967/jnumed.116.184028

8. Badawi RD, Shi H, Hu P, Chen S, Xu T, Price PM, et al. First human imaging studies with the EXPLORER total-body PET scanner. J Nucl Med. (2019) 60:299-303. doi: 10.2967/jnumed.119.226498
Ramsey Badawi at UC Davis. In formulating the projected clinical research and healthcare applications Total Body PET, the contribution is acknowledged of Pat Price in oncology along with many other clinical researchers in internal medicine.

9. Zhang X, Xie Z, Berg E, Judenhofer MS, Liu W, Xu T, et al. Total-body dynamic reconstruction and parametric imaging on the uEXPLORER. $J \mathrm{Nucl}$ Med. (2019) 61:285-91. doi: 10.2967/jnumed.119.230565

10. Karp JS, Vishwanath V, Geagan M, Muehllehner G, Pantel A, Parma M, et al. PennPET explorer: design and preliminary performance of a whole-body imager. J Nucl Med. (2020) 61:136-43. doi: 10.2967/jnumed.119.229997

11. Pantel AR, Viswanath V, Daube-Witherspoon ME, Dubroff JG, Muehllehner G, Parma MJ, et al. PennPET explorer: human imaging on a whole-body imager. J Nucl Med. (2020) 61:144-51. doi: 10.2967/jnumed.119.231845

Conflict of Interest: The University of California, Davis and United Imaging Healthcare have a research agreement and a revenue sharing agreement.

The author declares that the research was conducted in the absence of any commercial or financial relationships that could be construed as a potential conflict of interest.

Copyright (C) 2020 Jones. This is an open-access article distributed under the terms of the Creative Commons Attribution License (CC BY). The use, distribution or reproduction in other forums is permitted, provided the original author $(s)$ and the copyright owner(s) are credited and that the original publication in this journal is cited, in accordance with accepted academic practice. No use, distribution or reproduction is permitted which does not comply with these terms. 